\title{
Lateral Movement Method of Inverted Two-wheeled Vehicles for Narrow Roads Using Pivot Turn
}

\author{
Kazutaka Murakoshi* Non-member, Hironori Matsubara* Non-member \\ Yuki Nagatsu ${ }^{* a)} \quad$ Member, Hideki Hashimoto* Senior Member
}

(Manuscript received May 13, 2020, revised Oct. 2, 2020)

J-STAGE Advance published date : Dec. 4, 2020

\begin{abstract}
In recent years, the declining birthrate and an aging population have become a serious problem in Japan. For this reason, the number of elderly people who have difficulty going outside is expected to increase. In addition, elderly people have difficulty dealing with modes of transportation such as cars and bicycles. To solve these problems, a small and easy-to-handle mobile robot, such as an inverted two-wheel vehicle is expected to be a useful device. However, the vehicle robot has difficulty moving along a path with a narrower width than that of the vehicle body. Therefore, in this paper, a trajectory planning method is proposed that makes it possible for vehicles to move laterally on narrow roads using a pivot turns in places where it is otherwise difficult to turn. The validity of the proposed method was evaluated experimentally.
\end{abstract}

Keywords: inverted two wheels, pivot turn, trajectory tracking control

\section{Introduction}

In recent years, the declining birthrate and aging population have become problems in developed countries. According to the census of the Ministry of Internal Affairs and Communications in Japan, there are 15.78 million children 0-14 years in age in the country, corresponding to $12.4 \%$ of the total population. In addition, the proportion of the population aged 65 and over (aging rate) is $27.3 \%$, meaning that approximately 1 in 4 people in Japan are elderly.

In 2025, the aging rate will exceed $30 \%$, and in 2065 will reach $38.4 \%^{(1)}$. Therefore, because it is becoming increasingly difficult for elderly people to use transportation such as cars and bicycles, there are concerns about an increase number of "shopping refugees" among elderly people, and the burden placed on their movement has become a problem. In addition, in response to the declining labor force owing to the declining birthrate and aging population, there is also a need for robots to conduct tasks that have been performed by humans, and robots may be introduced for baggage transportation in factories and warehouses. To solve these problems, the use of small and easy-to-handle mobile robots, such as an inverted two-wheel vehicles, is expected.

Conventional inverted two-wheeled vehicles have the advantage of being able to turn on the spot and maintain the vehicle at a body level even on an inclined road surface compared to three- and four-wheeled vehicle robots. However, there is a problem in that the grounding point is only two wheels, creating static instability. In addition, there is a

\footnotetext{
a) Correspondence to: Yuki Nagatsu. E-mail: nagatsu@elect.chuou.ac.jp

${ }^{*}$ Department of Electrical Electronic, and Communication Engineering, Chuo University

1-13-27, Kasuga, Bunkyo-ku, Tokyo 112-8551, Japan
}

restriction in that an inverted two-wheel vehicle cannot move in the axle direction. For this reason, the vehicle robot has difficulty moving along a path that is narrower than the width of the vehicle body. On such a narrow path, the vehicle must move sideways while switching. However, an inverted twowheeled vehicle is unstable, as described above, and thus cannot drift.

Therefore, in this paper, a method is proposed that enables vehicles to move laterally on narrow roads that are difficult to sharply turn on. The proposed method uses pivot turning, which is applied in crawler-type moving mechanisms. Using the proposed method, a wheeled vehicle can easily move in the lateral direction without drifting. The proposed method is effective for narrow roads and corners that are difficult to navigate.

In conventional research, movements in all directions have been achieved using a complicated control mechanism, such as an omni-wheel. However, the method proposed in this paper does not require complicated mechanisms and can achieve lateral movement by combining simple turns. Therefore, one of the contributions of this paper is the realization of lateral movement for two-wheeled vehicles without using complicated mechanisms or the use of an Omni wheel.

In addition, in this study, the proposed method is evaluated from the viewpoint of the minimum jerk model. The results of the evaluation show that the proposed method contributes to lateral movements using a small jerking motion while satisfying the constraint conditions of the passage and maintaining a stable inverted motion.

\section{Modeling of Inverted Two-wheel Vehicle}

2.1 Inverted Two-wheel Vehicle Figure 1 shows an inverted two-wheel vehicle used in this paper. The inverted two-wheel vehicle measures $77.5 \mathrm{~cm}$ in height, $38 \mathrm{~cm}$ 


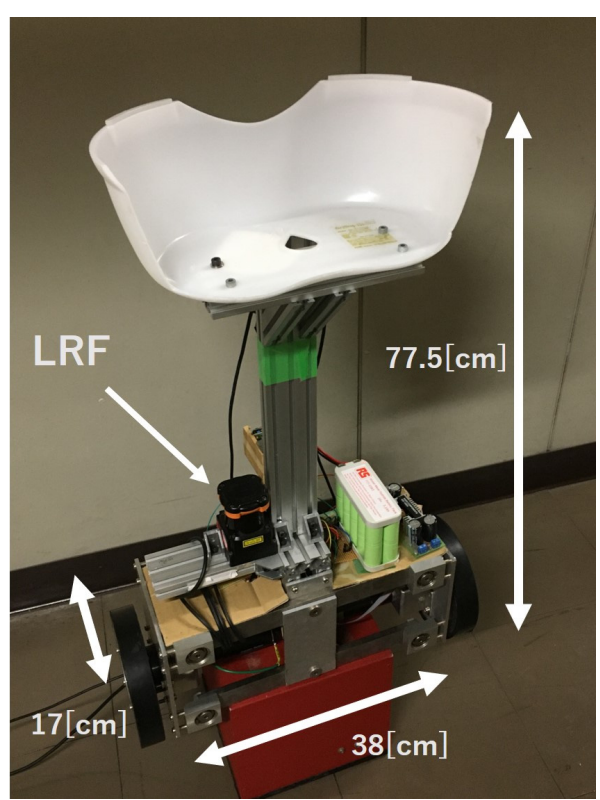

Fig. 1. Inverted two-wheel vehicle used in this paper

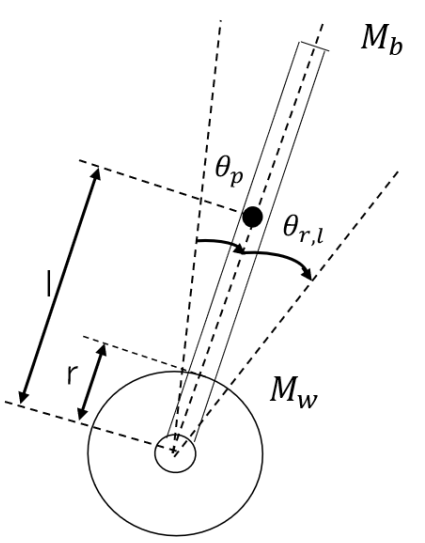

(a) Side view.

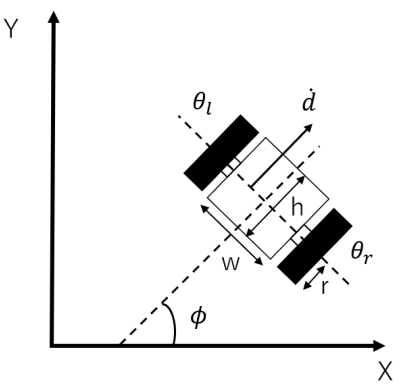

(b) Top view.
Fig. 2. Inverted two-wheel vehicle model

in width, and $17 \mathrm{~cm}$ in depth, and is equipped with two $90 \mathrm{~W}$ DC motors. The total weight of the car body is approximately $10.8 \mathrm{~kg}$. The model of the inverted two-wheel vehicle is shown in Fig. 2. The vehicle body tilt (hereinafter referred to as the attitude angle) $\theta_{p}$ of the inverted two-wheel vehicle is positive from the vertical direction. The wheel rotation angle $\theta_{r, l}$ is determined by the encoder attached to the motor. Calculated from the angle from the obtained vehicle body, the clockwise direction is positive, as is the attitude angle. The parameters of the inverted two-wheel vehicle are as listed in Table 1.

In deriving the kinematics of an inverted two-wheeled vehicle, two constraints are set such that the vehicle cannot move in the direction of the axle, and the left and right wheels do not slip. The first constraint can be expressed through the following equation:

$$
\dot{X} \sin \phi-\dot{Y} \cos \phi=0
$$

where $X$ and $Y$ represent the position of the inverted twowheel vehicle in the world coordinate system, respectively. Second, Eqs. (2) and (3) can be derived from the second constraint as follows ${ }^{(2)}$ :
Table 1. Inverted two-wheel vehicle parameters

\begin{tabular}{|c|c|c|}
\hline \multicolumn{2}{|r|}{ Parameter } & Value \\
\hline & : Sampling time & $1 \mathrm{~ms}$ \\
\hline$G$ & : Gravitational acceleration & $9.81 \mathrm{~m} / \mathrm{s}^{2}$ \\
\hline$r$ & : Wheel radius & $0.085 \mathrm{~m}$ \\
\hline$W$ & : Body width & $0.38 \mathrm{~m}$ \\
\hline$h$ & : Depth of body & $0.17 \mathrm{~m}$ \\
\hline$M_{w}$ & : Body mass & $9.4 \mathrm{~kg}$ \\
\hline$M_{b}$ & : Pendulum weight & $1.4 \mathrm{~kg}$ \\
\hline$l$ & : Center of gravity of body & $0.3 \mathrm{~m}$ \\
\hline$J_{y}$ & : Inertia around $\mathrm{Z}$ axis & $0.01332 \mathrm{kgm}^{2}$ \\
\hline$W_{r}$ & : pivot turn radius & $0.22 \mathrm{~m}$ \\
\hline$X_{R, L}$ & : Left and right wheel position $\mathrm{X}$ coordinate & \\
\hline$Y_{R, L}$ & : Left and right wheel position $\mathrm{Y}$ coordinate & \\
\hline$d$ & : Translation angle & \\
\hline$\phi$ & : Turning angle & \\
\hline$X, Y$ & : Body position coordinates & \\
\hline
\end{tabular}

$$
\begin{aligned}
& \dot{X} \cos \phi-\dot{Y} \sin \phi+\frac{W}{2} \dot{\phi}=r \dot{\theta}_{r} . \\
& \dot{X} \cos \phi-\dot{Y} \sin \phi-\frac{W}{2} \dot{\phi}=r \dot{\theta}_{l} .
\end{aligned}
$$

Based on the Lagrangian equation, the equation of motion for the inverted two-wheel vehicle in the wheel space is expressed as follows:

$$
\begin{aligned}
\boldsymbol{J}(\boldsymbol{q}) \ddot{\boldsymbol{q}} & =\boldsymbol{\tau}^{\mathrm{cmd}}-\boldsymbol{\tau}^{\mathrm{ext}}-\boldsymbol{h}(\boldsymbol{q}, \dot{\boldsymbol{q}})-\boldsymbol{g}(\boldsymbol{q}) \\
q & =\left[\begin{array}{lll}
\theta_{p} & \theta_{r} & \theta_{l}
\end{array}\right]^{T} \ldots \ldots \ldots \ldots \ldots \\
\boldsymbol{\tau}^{\mathrm{ref}} & =\left[\begin{array}{lll}
0 & \tau_{r}^{\mathrm{ref}} & \tau_{l}^{\mathrm{ref}}
\end{array}\right]^{T} \ldots \ldots \ldots \ldots \ldots \\
\boldsymbol{\tau}^{\mathrm{ext}} & =\left[\begin{array}{lll}
0 & \tau_{r}^{\mathrm{ext}} & \tau_{l}^{\mathrm{ext}}
\end{array}\right]^{T} \ldots \ldots \ldots \ldots
\end{aligned}
$$

Here, $\boldsymbol{q}, \boldsymbol{\tau}, \boldsymbol{J}(\boldsymbol{q}), \boldsymbol{h}(\boldsymbol{q}, \dot{\boldsymbol{q}})$ and $\boldsymbol{g}(\boldsymbol{q})$ represent the wheel space angle vector, torque vector, inertia matrix, Coriolis term, and gravity vector, respectively. In addition, suffix res, ref, and ext represent the response value, reference value, and external force term, respectively.

The kinematic relationship between the wheel space of the inverted two-wheel vehicle and the translation/turning direction can be expressed as follows using the Jacobian matrix $\boldsymbol{J}_{\text {aco }}$ from the constraints of Eqs. (2) and (3).

$$
\dot{\boldsymbol{x}}=\left[\begin{array}{c}
\dot{\theta}_{p} \\
\dot{d} \\
\dot{\phi}
\end{array}\right]=\left[\begin{array}{ccc}
1 & 0 & 0 \\
0 & \frac{R}{2} & \frac{R}{2} \\
0 & \frac{R}{W} & -\frac{R}{W}
\end{array}\right]\left[\begin{array}{c}
\dot{\theta}_{p} \\
\dot{\theta}_{r} \\
\dot{\theta}_{l}
\end{array}\right]=\boldsymbol{J}_{\mathrm{aco}} \dot{\boldsymbol{q}} \cdots \ldots \ldots
$$

In this research, it is necessary to make the vehicle follow the target trajectory, and thus the position of the vehicle is acquired and controlled. Therefore, the kinematic relationship between the translation/turning direction of the inverted two-wheel vehicle and the space in-state $Q$ is expressed by the following equation using the position coordinate vector $\boldsymbol{X}$ and matrix $\boldsymbol{T}^{(3)}$ :

$$
\dot{\boldsymbol{Q}}=\left[\begin{array}{c}
\dot{\theta}_{p} \\
\dot{X} \\
\dot{Y} \\
\dot{\phi}
\end{array}\right]=\left[\begin{array}{c}
\dot{\theta}_{p} \\
\dot{\boldsymbol{X}}
\end{array}\right]=\left[\begin{array}{ccc}
1 & 0 & 0 \\
0 & \cos \phi & 0 \\
0 & \sin \phi & 0 \\
0 & 0 & 1
\end{array}\right]\left[\begin{array}{c}
\dot{\theta}_{p} \\
\dot{d} \\
\dot{\phi}
\end{array}\right]=\boldsymbol{T} \dot{\boldsymbol{X}} \cdots \cdots
$$

In addition, for force $f$ in the translational/turning space and force $\boldsymbol{F}$ in the space in state $\boldsymbol{Q}$, the relational expression in Eq. (10) is established using the pseudo-inverse matrix $\boldsymbol{T}^{+T}$. 


$$
\boldsymbol{F}=\boldsymbol{T}^{+T} \boldsymbol{f}
$$

From Eqs. (9) and (10), the motion equation in the wheel space can be transformed into the motion equation of the space in state $\boldsymbol{Q}$ as follows:

$$
\boldsymbol{M} \ddot{\boldsymbol{Q}}=\boldsymbol{F}^{\mathrm{ref}}-\boldsymbol{F}^{\mathrm{ext}}-\boldsymbol{H}(\boldsymbol{Q}, \dot{Q})-\boldsymbol{G}(\boldsymbol{Q})
$$

where $\boldsymbol{F}, \boldsymbol{M}, \boldsymbol{H}(\boldsymbol{Q}, \dot{\boldsymbol{Q}})$ and $\boldsymbol{G}(\boldsymbol{Q})$ represents the force vector, equivalent mass matrix, Coriolis term, and gravity term at $\boldsymbol{Q}$.

\section{Proposed Method}

3.1 Pivot Turn An inverted two-wheeled vehicle has difficulty moving along a path that has a narrower width than that of the vehicle body. It is therefore necessary to travel while drifting or switching to the vehicle body. However, because of a static instability, conventional drifting is difficult to achieve for an inverted two-wheeled vehicle.

Therefore, in this study, lateral movement is applied using a pivot turn. The method of travel using a pivot turn is used in a wheel- or crawler-type moving mechanism. During a pivot turn, the movement of either the left or right wheel is stopped and the other wheel is driven ${ }^{(4)}$. That is, a turning motion is applied by using the center of the wheel when placed at the center of the vehicle as a radius.

3.2 Lateral Movement Method using Pivot Turn The proposed method considers lateral movement using the pivot turn mentioned above. As shown in Fig. 3, the inverted two-wheeled vehicle is assumed to be in a passage between walls, which is narrower than the vehicle body width. Because of this, it is difficult to run in the axle direction because it cannot turn on the spot. Therefore, the inverted two-wheel vehicle moves laterally while switching back, according to the following procedure.

(1) Only the left wheel moves forward to a certain turning angle.

(2) Only the right wheel is advanced to keep the vehicle level.

(3) Only the left wheel moves backward to a certain turning angle.

(4) Only the left wheel is receded to keep the vehicle level.

By repeating the above procedure, the inverted two-wheeled vehicle can travel in the lateral direction. As a result, it is necessary to use at least four pivots to move in the lateral

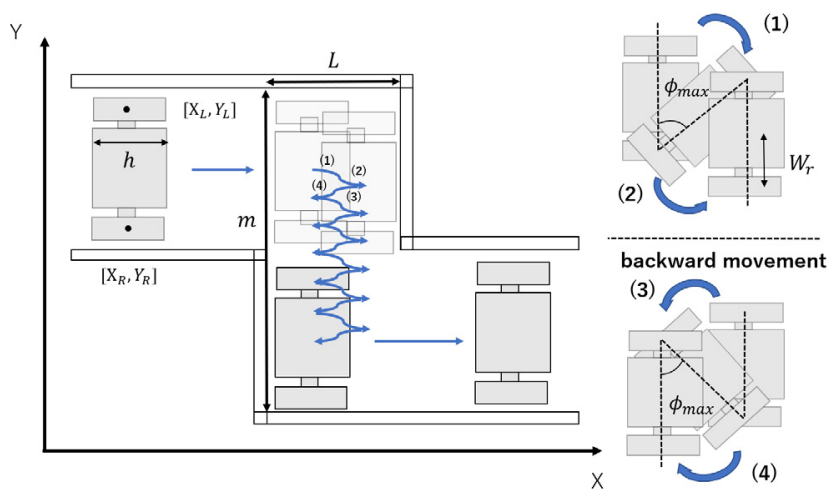

Fig. 3. Lateral movement using a pivot turn direction ${ }^{(5)}$. When the inverted two-wheel vehicle moves forward with lateral motion, as shown in Fig. 3, the trajectory of lateral movement using the pivot turn is expressed using position $[X, Y]$ as follows:

$$
\begin{aligned}
& X_{1}=X_{R 1}+W_{r} \sin \phi_{1}^{c m d}(t) \\
& Y_{1}=Y_{R 1}+W_{r} \cos \phi_{1}^{c m d}(t) \\
& X_{2}=X_{L 2}-W_{r} \sin \phi_{2}^{c m d}(t) \\
& Y_{2}=Y_{L 2}-W_{r} \cos \phi_{2}^{c m d}(t) \cdots
\end{aligned}
$$

In the case of backward movement, it can be expressed as follows:

$$
\begin{aligned}
X_{3} & =X_{R 3}-W_{r} \sin \phi_{3}^{c m d}(t) \\
Y_{3} & =Y_{R 3}+W_{r} \cos \phi_{3}^{c m d}(t) \\
X_{4} & =X_{L 4}+W_{r} \sin \phi_{4}^{c m d}(t) \\
Y_{4} & =Y_{L 4}-W_{r} \cos \phi_{4}^{c m d}(t)
\end{aligned}
$$

where

$$
\begin{aligned}
\phi_{i}^{c m d} & =\phi_{\max } \frac{t-i}{T / 4}(i: \text { odd number }) \cdots \ldots \ldots . . \\
\phi_{j}^{c m d} & =\phi_{\max }-\phi_{\max } \frac{t-j}{T / 4}(j: \text { even number }) .
\end{aligned}
$$

In Eqs. (16) and (17), $T$ represents the time until the forward and backward movements are completed. In this study, $\phi_{\max }$ and $T$ are set to $0.2527 \mathrm{rad}$ and $4 \mathrm{~s}$, respectively. In Eqs. (12)(15), $X_{R}, X_{L}, Y_{R}, Y_{L}$, and $W_{r}$ are the center coordinates of the left and right wheels at the pivot turn motion, and the radius of the pivot turn, respectively.

In addition, the maximum turning angle of the pivot turn is derived from Eq. (12) as follows:

$$
\phi_{\max }=\arcsin \left[\frac{(L-h)-X_{R 1}}{2 W_{r}}\right] \text {. }
$$

Here, $L$ and $h$ are the widths of the traveling path and the width of the vehicle body, respectively

In the case of a pivot turn, the vehicle turns to the maximum turning angle $\phi_{\max }$. That is, the maximum turning angle of the pivot turn depends on the width of the traveling path and the width of the vehicle body. Therefore, when a lateral movement using a pivot turn is applied, the distance between the traveling narrow road is $m$, and the relationship with the number of times $n$ that the inverted two-wheel vehicle turns over can be expressed through the following equations derived from Eq. (18):

$$
\begin{aligned}
n & =\frac{m}{Y_{L 4}-W_{r} \cos \phi_{4}^{c m d}} \cdots \ldots \ldots \ldots \ldots \\
& =\frac{m}{Y_{L 4}-W_{r} \cos \left(\arcsin \left[\frac{(L-h)-X_{R 1}}{2 W_{r}}\right]\right)} \cdots \\
& =\frac{m}{Y_{L 4}-W_{r} \sqrt{1-\left(\frac{(L-h)-X_{R 1}}{2 W_{r}}\right)^{2}}} \cdots \cdots
\end{aligned}
$$

From Eq. (21), the number $n$ of this lateral movement using a pivot turn depends on the maximum turning angle. That is, it depends on the width of the path. Accordingly, the larger the maximum turning angle, the larger the distance traveled when turning once in the axle direction, and the number of turns decreases. 
3.3 Evaluation of Lateral Move Trajectory using the Minimum Jerk Model In this section, lateral trajectories using the pivot turn of the proposed method are evaluated. As an evaluation, an inverted two-wheeled vehicle is assumed to move sideways, as shown in Fig. 3. In addition, the vehicle switches toward in the $\mathrm{X}$-axis direction and moves in the $\mathrm{Y}$ axis direction. Therefore, the position of $Y(t)$ in the trajectory of the vehicle is evaluated.

The minimum jerk model was used to evaluate the trajectory $Y(t)$. The minimum jerk criterion is proposed as a model for expressing voluntary movements of human basic movements such as reaching movements in the research of movement functions of living bodies ${ }^{(6)}$. For the position trajectory $Y(t)$, the evaluation function $C_{j}$ expressed by the following equation is minimized:

$$
C_{j}(t)=\int_{0}^{t_{f}}\left(\frac{d^{3} Y(t)}{d t^{3}}\right)^{2} d t \ldots \ldots \ldots \ldots \ldots \ldots \ldots
$$

Here, $t_{f}$ is the time to the endpoint. By using the minimum jerk model to evaluate the position trajectory, it is possible to evaluate whether the trajectory can or cannot suppress the jerk. In other words because it is possible to judge whether the ratio of variation in the acceleration is suppressed, it is possible to evaluate whether the vehicle can move laterally while maintaining the stability of the vehicle body position. A position trajectory $Y(t)$ is generated between start point $Y_{0}$ and endpoint $Y_{f}$. In addition, the evaluation function $C_{j}$ of Eq. (22) takes an extreme value when $Y(t)$ satisfies the EulerPoisson equation. If the value is obtained, the following conditional equation can be derived:

$$
\frac{d^{6} Y(t)}{d t^{6}}=0
$$

From Eq. (23), $Y(t)$ can be expressed by a fifth-order polynomial. Therefore, if the velocity and acceleration at the start point $Y_{0}$ and endpoint $Y_{f}$ are assumed to be zero, the position trajectory $y(t)$ can be expressed by the following equation based on the minimum jerk model. The boundary condition number is $6^{(7)}$.

$$
\begin{aligned}
Y(\tau) & =Y_{0}+\left(Y_{f}-Y_{0}\right)\left(6 \tau^{5}-15 \tau^{4}+10 \tau^{3}\right) \ldots \ldots \ldots \\
\tau & =\frac{t}{t_{f}} \ldots \ldots \ldots \ldots \ldots \ldots \ldots \ldots \ldots \ldots \ldots \ldots \ldots \ldots \ldots \ldots
\end{aligned}
$$

In this study, the trajectory generated by Eq. (24) is called the minimum jerk trajectory.

This research compares the three trajectories: the minimum jerk trajectory, the shortest movement path between two points, and the proposed method based on a pivot turn. Because the vehicle is assumed to travel in a narrow path parallel to the Y-axis, this study uses and evaluates the minimum jerk trajectory only in the Y-axis direction. With the minimum jerk trajectory, it is difficult to generate a trajectory considering restrictions such as the size of the vehicle body. Therefore, the trajectory for the shortest travel path is applied to the $\mathrm{X}$-axis of the method using the minimum jerk degree trajectory in the Y-axis. A comparison of each orbit is shown in Fig. 4 , and the result derived by the evaluation function $C_{j}$ for each trajectory is shown in Fig. 5 .

From Fig. 4, the pivot turn of the proposed method has a

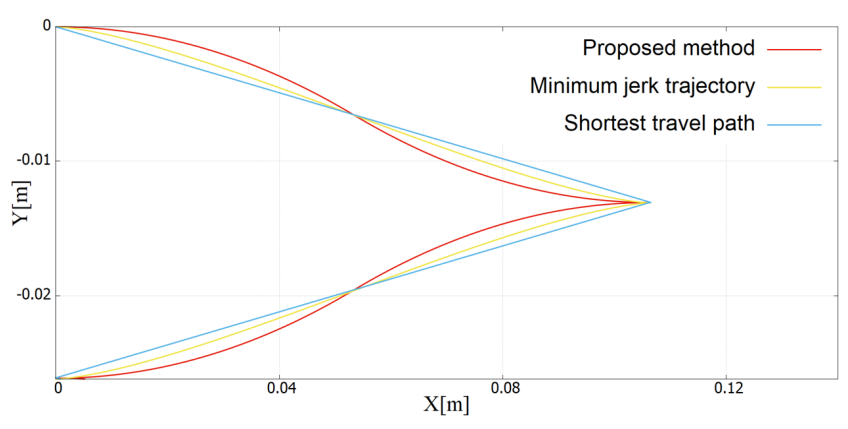

Fig. 4. Comparison of trajectory

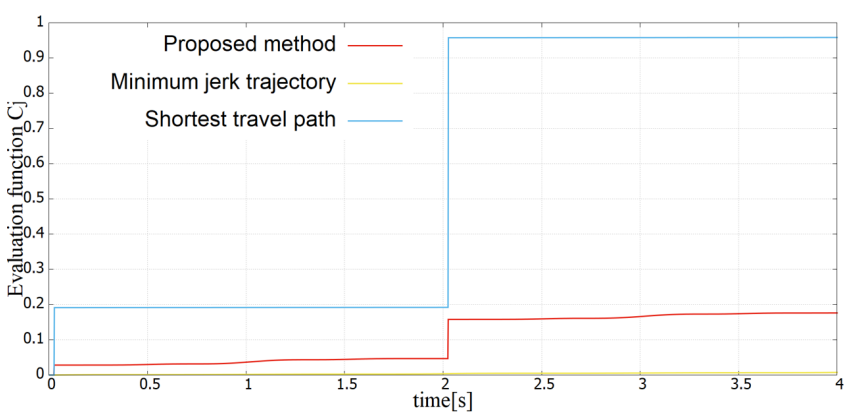

Fig. 5. Comparison of evaluation function $C_{j}$

longer moving distance than the triangular trajectory, which is the shortest travel path. It is also confirmed that the minimum trajectory is a trajectory that passes between the trajectory of the proposed method and the triangular wave, which is the shortest travel path. When each trajectory is evaluated from Fig. 5 with respect to evaluation function $C_{j}$, the value of the proposed trajectory is approximately 10-times larger than that of the minimum trajectory. However, the value of the shortest travel path is approximately 50-times larger than that of the minimum trajectory. In other words, the value of the proposed method is approximately 5 -times smaller than that of the shortest travel path. Therefore, it can be confirmed that the evaluation function of the proposed method has a smaller evaluation function than the shortest travel path. Accordingly, it was shown that the pivot turn of the proposed method is a track close to the minimum jerk trajectory and can smoothly travel in the lateral direction from the shortest travel path.

In addition, the trajectory of the proposed method has an advantage in that the trajectory can be derived from the width of the passage from Eq. (21). Therefore, it is possible to set an area where an inverted two-wheeled vehicle can move without hitting a wall along a narrow path, allowing a stable and lateral motion to be achieved. By contrast, the minimum jerk trajectory must have its start and endpoints set individually. It is difficult to derive the two points without the vehicle hitting the wall in a narrow path. For this reason, with the proposed method, the track can travel laterally along a narrow path without hitting the wall in comparison to the minimum jerk trajectory. As a result, the trajectory of the proposed method is considered to be more suitable for a lateral movement along a narrow path than the other trajectories and is used for the experiments conducted in this research.

3.4 Body Position Conditions When traveling along a narrow road, it is necessary to acquire the position of the 


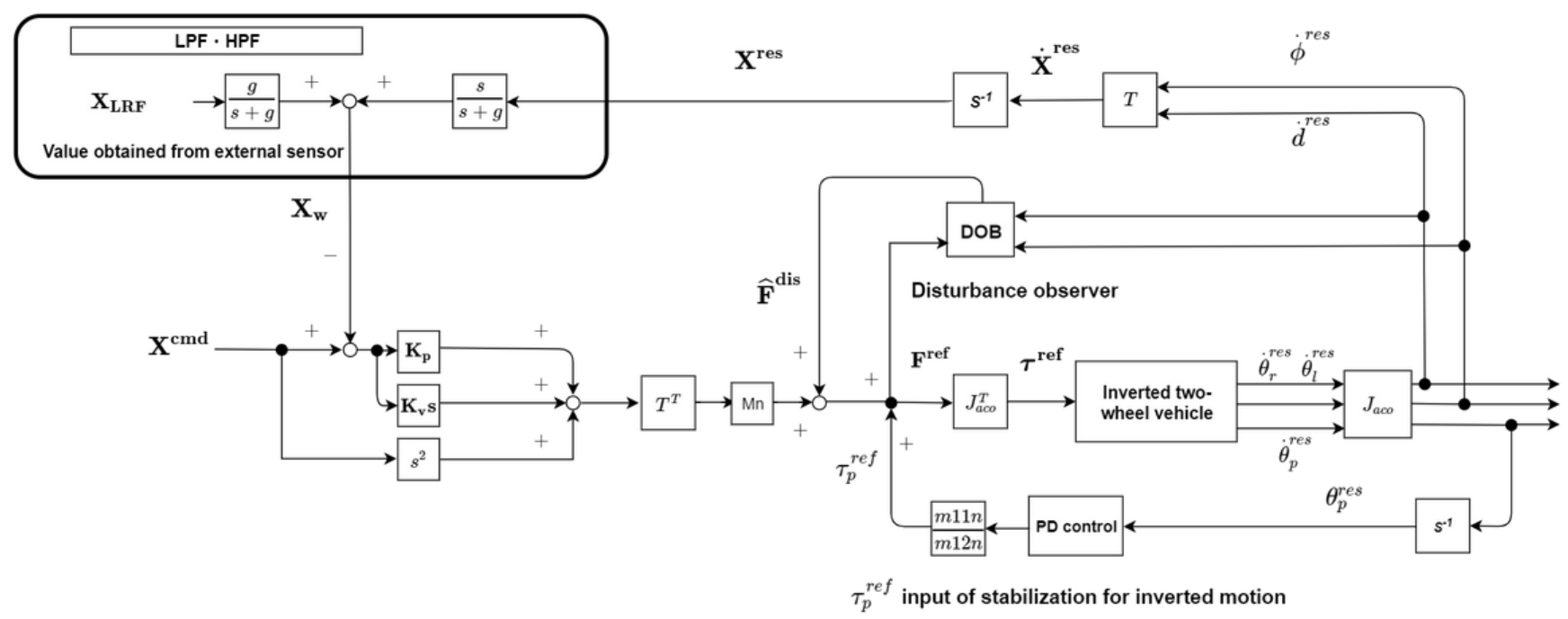

Fig. 6. Block diagram for narrow road traveling

vehicle body. To acquire the position of the body, odometry measurements using an encoder in an inverted two-wheel vehicle, that is, a dead reckoning, are used. Self-localization is often used for autonomous mobile robots ${ }^{(8)}$. However, with dead reckoning alone, errors accumulate over time owing to changes in the wheel radius resulting from irregularities on the road surface and the resolution of the encoder ${ }^{(9)}$. As a result, the position of the vehicle shifts, and it becomes difficult to travel on a narrow road, with the vehicle hitting a wall among other problems. It is therefore necessary to use an external sensor along with an internal sensor such as an encoder.

In this study, a laser range finder (LRF), which is often used as an external sensor, is applied. In recent years, the use of such LRFs has become widespread in the field of mobile robots. The measurement principle is to irradiate the laser from the LRF and calculate the distance to the object based on the time until it reflects and returns from the object. The URF-30LX is used as the LRF, and the parameters are a scan time of $25 \mathrm{~ms}$ and a ranging resolution of $1 \mathrm{~mm}$. The LRF was set up at a height of $25 \mathrm{~cm}$ from the ground, as shown in Fig. 1, with a $6.5 \mathrm{~cm}$ shift in the $\mathrm{Y}$ direction from the center of the vehicle body.

However, measurement errors of the LRF caused by noise accumulate over time, and thus it is necessary to remove the noise. Therefore, this research uses both the odometry $\boldsymbol{X}^{\text {res }}$ obtained from the encoder attached to the vehicle wheel, and the value $\boldsymbol{X}_{L R F}$ obtained from the LRF. In addition, $\boldsymbol{X}^{r e s}$ and $\boldsymbol{X}_{L R F}$ are passed through a high-pass filter and a low-pass filter, respectively. Thus, the position $\boldsymbol{X}_{w}$ of the vehicle in the absolute coordinate system can be expressed by the following equation using the position value obtained from each filter:

$$
\boldsymbol{X}_{w}=\frac{g}{s+g} \boldsymbol{X}_{L R F}+\frac{s}{s+g} \boldsymbol{X}^{r e s} \ldots \ldots \ldots \ldots \ldots \ldots
$$

The LRF is connected to an external PC and uses the ROS to read the distance information. The PC calculates the robot position $\boldsymbol{X}_{\boldsymbol{L} \boldsymbol{R} \boldsymbol{F}}$ from the read distance information obtained by the LRF. The robot position is transmitted to the controller through a serial communication, and the self-position is estimated using Eq. (26). Together with the target position trajec- tory, the value obtained from Eq. (26) is used as the position response of the vehicle for position control.

3.5 Controller Design The controller design used in this research is described. The inverted two-wheel vehicle travels to the target position while maintaining an inverted state. Therefore, it is necessary to achieve both inverted control and position control. PD control was applied to translational/turning and inverted control. In addition, position control in state $\boldsymbol{Q}$ based on the acceleration control is applied using the acceleration reference value in the following equation:

$$
\ddot{\boldsymbol{Q}}^{r e f}=\ddot{\boldsymbol{Q}}^{c m d}+\boldsymbol{K}_{\boldsymbol{v}}\left(\dot{\boldsymbol{Q}}^{c m d}-\dot{\boldsymbol{Q}}\right)+\boldsymbol{K}_{\boldsymbol{p}}\left(\boldsymbol{Q}^{c m d}-\boldsymbol{Q}\right) \cdots \cdots
$$

Here, $\boldsymbol{X}^{\text {cmd }}, \boldsymbol{K}_{\boldsymbol{v}}$, and $\boldsymbol{K}_{\boldsymbol{p}}$, represent a command value, velocity gain, and position gain, respectively. By using Eq. (27), the inverted two-wheeled vehicle can follow the position, velocity, and acceleration of the target trajectory while maintaining a stable posture. The gain of each control was determined by trial and error using the limit sensitivity method.

Furthermore, in this research, a disturbance observer $(\mathrm{DOB})^{(2)}$ is used in the translational and swirling spaces to reduce the influence of disturbances such as friction and modeling errors and increase the robustness. The disturbance observer estimates and compensates the centrifugal/Coriolis force term, gravity term, external force term, and viscous friction term. The disturbance observer estimates and compensates the centrifugal/Coriolis force term, gravity term, external force term, and viscous friction term.

$$
\hat{\boldsymbol{F}}^{\text {dis }}=\left[\begin{array}{lll}
0 & \hat{f}_{d}^{d i s} & \hat{\tau}_{\phi}^{d i s}
\end{array}\right]^{T} \ldots
$$

By adding Eq. (28) to the reference torque, the influence of the disturbance can be reduced. A block diagram of the entire vehicle, including the control design, is shown in Fig. 6. Here, $\boldsymbol{M}_{\boldsymbol{n}}, m 11 n$, and $m 12 n$ are the nominal mass matrices with only the diagonal elements, the first row and first column element, and the first row and second column element of the nominal mass matrix, respectively.

The cutoff frequency of the disturbance observer is determined to be as high as possible within the range where the noise does not significantly affect the estimated disturbance. 
In addition, the cutoff frequency of the filter used to estimate $\boldsymbol{X}_{\boldsymbol{w}}$ is determined by trial and error.

\section{Simulation for Evaluation of Body Angle}

In this study, the variation in body angle is evaluated based on simulations. In the simulations, traveling motions with three trajectories are applied: the minimum jerk trajectory, the shortest movement path between two points, and the proposed method based on a pivot turn. The parameters for the controllers in the simulations of each method are set such that the method using each trajectory can realize a stable inverted motion.

Figure 7 shows the simulation results for the body angle. It is also confirmed that the body angle of the proposed method sways by approximately one-fifth that of the method using the shortest path trajectory. In addition, the results show that the body angle of the proposed method sways by approximately half as much as that of the method using the minimum jerk trajectory in the Y-axis direction. Therefore, the proposed method has an advantage in terms of the stability of the inverted motion, which is affected by interference from the translational direction.

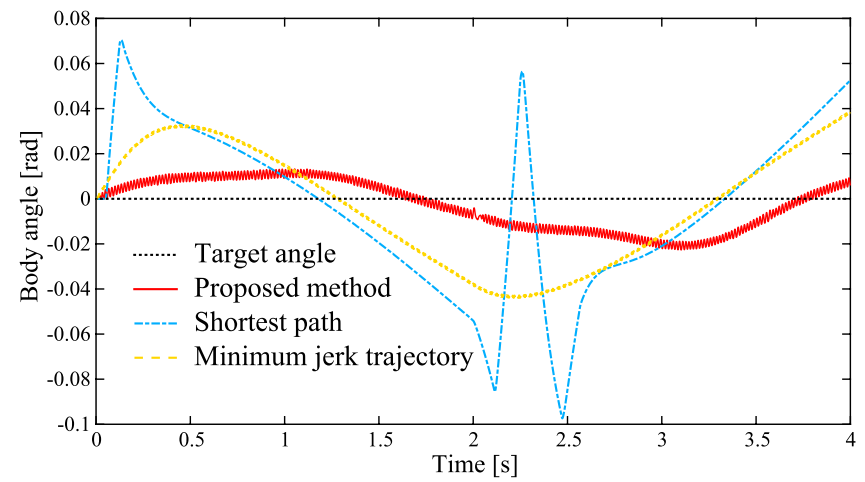

Fig. 7. Simulation results of body angle

\section{Experiments}

5.1 Experimental Conditions This section describes experiments on actual machines conducted to verify the effectiveness of the proposed method. The initial position $\left[X^{r e s}, Y^{r e s}\right]$ of the inverted two-wheel vehicle is $[0,0]$, the initial turning angle $\phi^{\text {res }}$ is $0 \mathrm{rad}$, and the width of the path to the corner is $0.5 \mathrm{~m}$. Therefore, the length $L$ of the passage for lateral movement is set to $0.3 \mathrm{~m}$ with reference to the size of a Rumba cleaning robot. A wall was created by assuming a narrow corner street, and the above conditions were included. The experimental environment created in this study is shown in Fig. 8. As shown in Fig. 8, the axle direction movement is performed in the downward direction shown in the figure, with the initial position before the corner. At that time, the actual running state was photographed using a $120^{\circ}$ camera lens.

5.2 Experiments Results The state of the lateral movement tested on a narrow road is shown in Fig. 9. The results obtained are shown in Figs. 10, and 11. From Fig. 10, it can be observed that the inverted two-wheeled vehicle follows the target trajectory. In addition, because it is traveling in a movable area, the inverted two-wheeled vehicle can move laterally without hitting the wall.

From Fig. 11, it was found that the posture of the vehicle tilts forward when the vehicle body moves forward. In addi-

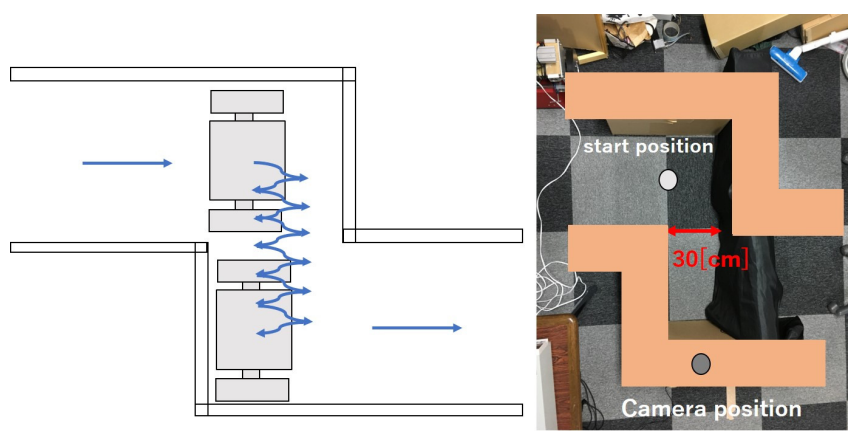

Fig. 8. Experiment environment

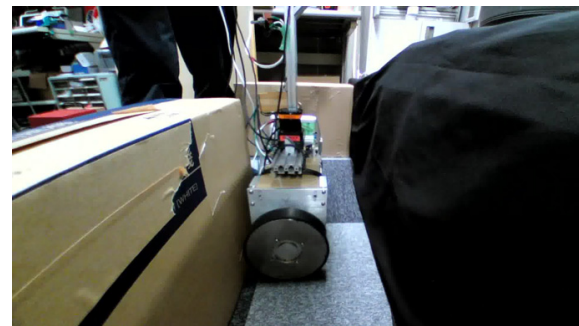

(a) Start.

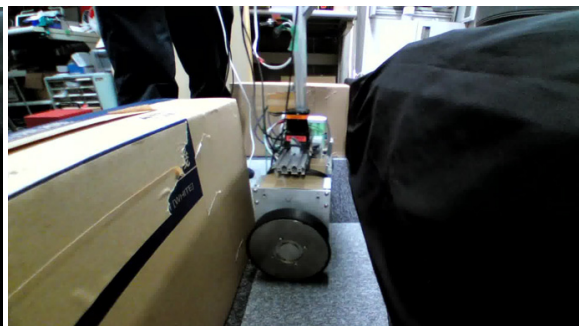

(b) First pivot turn

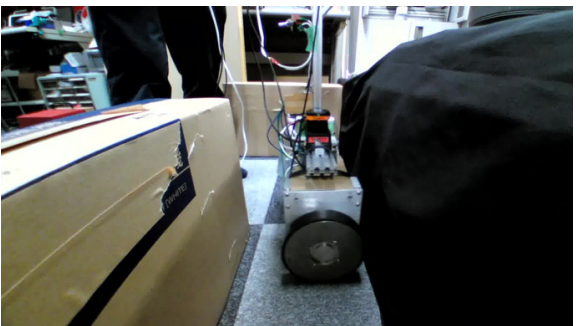

(c) Second pivot turn.

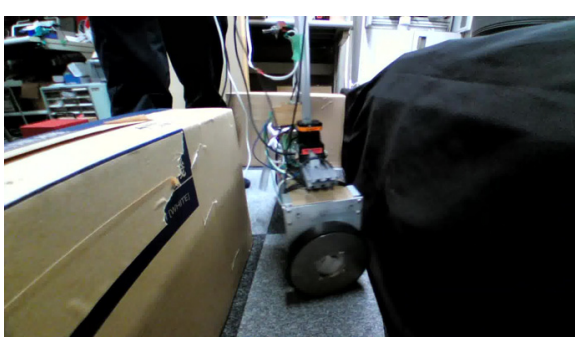

(d) Third pivot turn

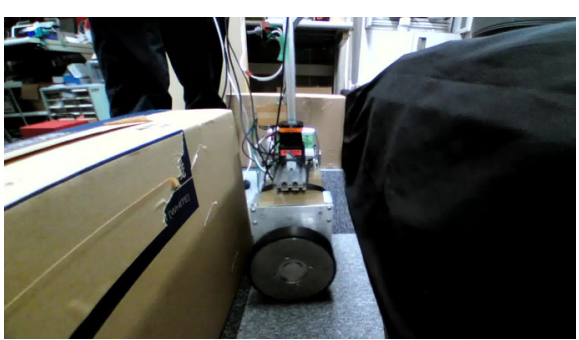

(e) Fourth pivot turn.

Fig. 9. Sideways movement of an inverted two-wheeled vehicle 


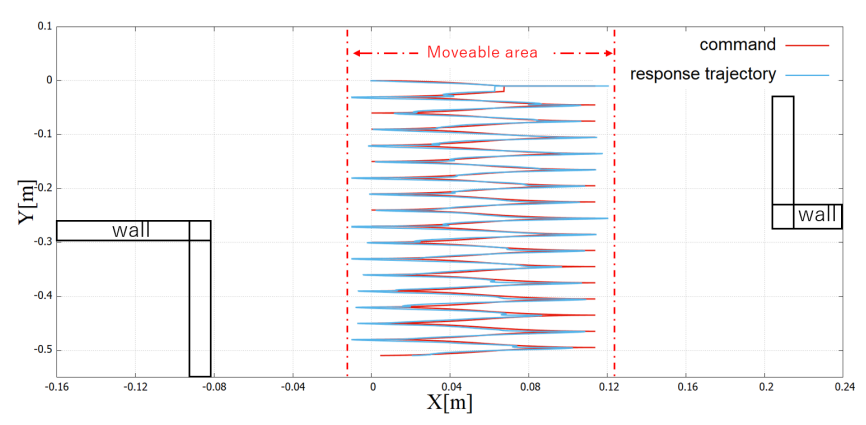

Fig. 10. Results of body position

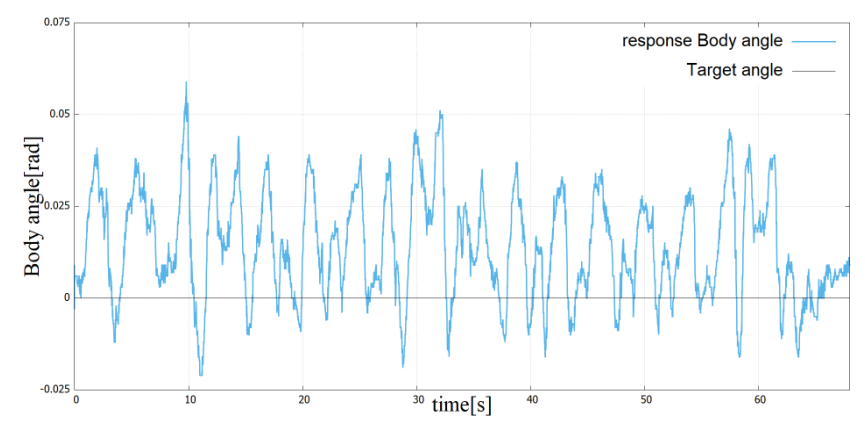

Fig. 11. Result of body angle

tion, the posture tilts backward when the body moves backward. Therefore, it does not converge to the target posture angle 0 rad during lateral movement and swings back and forth while constrained within the target posture angle. However, it can be confirmed that the inverted two-wheeled vehicle operates stably while maintaining the attitude angle without becoming unstable. In addition, the attitude angle swings by a maximum of $0.059 \mathrm{rad}$ and tilts by approximately $3.4^{\circ}$. The attitude angle is small and does not affect the operation. Therefore, an inverted two-wheel vehicle can be operated using the proposed method while achieving both inverted and positional control.

When traveling on a narrow road by an inverted twowheeled vehicle, it is necessary to travel without hitting the walls or curb blocks and without destabilization. Therefore, the amount of protrusion in the $\mathrm{X}$-axis direction must be as small as possible. The shortest path with a linear trajectory can be considered as one such option. However, the shortest trajectory induces a large overshoot in the pitch angle when the vehicle switches between the forward and backward directions under increased interference from the vehicle body motion, as shown in the simulation results. As for the trajectory with the minimum jerking, it is difficult to design the trajectory by considering the constraints of the passage width. By contrast, the proposed method makes a lateral movement possible with smaller jerks than that of the shortest path trajectory while satisfying the constraint conditions of the passage and maintaining a stable inverted motion. Thus, the technique used by the proposed system for the control of lateral movement is suitable for an inverted two-wheel vehicle and satisfies the required performance.

From the above, it is confirmed that the lateral movement method using the proposed method can stably move laterally without colliding with a wall even in narrow roads.

\section{Conclusions}

In this study, a lateral movement method for an inverted two-wheeled vehicle that can operate on a narrow road is proposed. A conventional inverted two-wheeled vehicle has only two wheels on the ground and is statically unstable, and thus drifting is difficult to achieve with a conventional method, making it is difficult to travel in a passage narrower than the width of the vehicle body. Therefore, a trajectory was generated using a pivot turn to enable lateral movement. Based on the minimum jerk model, the proposed method has less jerking than the shortest travel path. In addition, the results of the experiment using an actual inverted two-wheel vehicle showed that the ability to move sideways while achieving both inversion and position control, and the ability to move on narrow roads with conventional traveling paths, are difficult to achieve.

In the future, human cooperative motions of an inverted two-wheeled vehicle using force control in combination with the pivot turn approach proposed in this research will be investigated.

\section{Acknowledgment}

This work was supported in part by KEIRIN JKA (2020M188).

\section{References}

( 1 ) Y. Nagatsu, Y. Matsushita, K. Murakoshi, and H. Hashimoto: "Switching Control of Personal Vehicle with Two wheels and Three Wheels Modes", IEEJ International Workshop on Sensing, Actuation Control, and Optimization (2019)

( 2 ) T. Kawamura and T. Murakami: "Multifunctional Control of a Two-Wheel Driven Wheelchair Considering Comfort of a Passenger", IEEJ Transactions on Industry Applications, Vol.133, No.4, pp.404-413 (2013)

( 3 ) A. Nakamura and T. Murakami: "A Stabilization Control of Two Wheels Driven Wheelchair", 2009 IEEE/RSJ International Conference on Intelligent Robots and Systems, pp.4863-4868 (2009)

( 4 ) M. Ichikawa: "Wheel Arrangements for Wheeled Vehicle", Journal of the Robotics Society of Japan, Vol.13, No.1, pp.107-112 (1995)

( 5 ) K. Murakoshi, Y. Matsubara, Y. Nagatsu, and H. Hashimoto: "Examination of lateral movement method in inverted two-wheel vehicle", The 37th Annual Conference of the Robotics Society of Japan (2019)

( 6 ) T. Flash and N. Hogan: "The coordination of arm movements: an experimentally confirmed mathematical model”, Journal of Neuroscience, Vol.5, No.7, pp.1688-1703 (1985)

( 7 ) H. Seki, T. Sugimoto, and S. Tadakuma: "Novel Driving Control of Power Assisted Wheelchair Based on Minimum Jerk Trajectory", IEEJ Transactions on Electronics, Information and Systems, Vol.125, No.7, pp.1133-1139 (2005)

( 8 ) H. Nakagomi and Y. Fuse: "Development of Crawler Robot System for Autonomous Mapping", Yamanashi Prefectural Industrial Technology Center research report, No.30, pp.27-29 (2016)

( 9 ) M. Hashimoto, F. Oba, Y. Fujikawa, K. Imamaki, and T. Nishida: "Position Estimation Method for Wheeled Mobile Robot by Integrating Laser Navigation and Dead Reckoning Systems", Journal of the Robotics Society of Japan, Vol.11, No.7, pp.1028-1038 (1993)

Kazutaka Murakoshi (Non-member) received the B.E. and M.E. de-

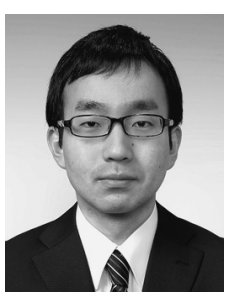
grees in electrical, electronic, and communication engineering from Chuo University, Tokyo, Japan, in 2018 and 2020, respectively. His research interests include motion control, mobile robots, and intelligent space. 
Hironori Matsubara (Non-member) received the B.E. degree in elec-

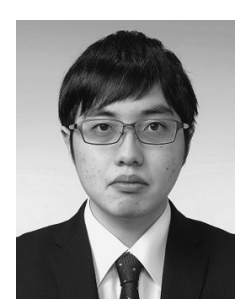
trical, electronic, and communication engineering from Chuo University, Tokyo, Japan, in 2019. He is currently working toward the M.E. degree in electrical, electronic, and communication engineering at the postgraduate program of the Chuo University. His research interests include motion control, robotics, and intelligent space.

Yuki Nagatsu

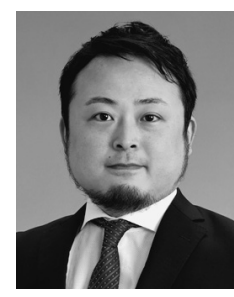

(Member) received the B.E. degree in system design engineering and the M.E. and Ph.D. in integrated design engineering from Keio University, Yokohama, Japan, in 2012, 2014 and 2017, respectively. Since 2017, he has been with the Department of Electrical, Electronic, and Communication Engineering, Chuo University, Tokyo, Japan. Currently, he is working as an Assistant Professor. His research interests include motion control, robotics, real-world haptics, intelligent space and human support systems. He is a Member of the Institute of Electrical and Electronics Engineers (IEEE), the Robotics Society of Japan (RSJ), and the Japan Society for Precision Engineering (JSPE).
Hideki Hashimoto (Senior Member) received the B.E., M.E., and

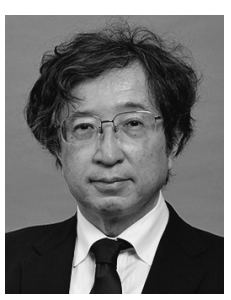
Dr. Eng. degrees in electrical engineering from the University of Tokyo, Tokyo, Japan, in 1981, 1984, and 1987, respectively. He joined the Institute of Industrial Science, University of Tokyo, as a Lecturer in 1987, and was an Associate Professor from 1990 to 2010. He moved to Chuo University, Tokyo, as a Professor in 2011. From 1989 to 1990, he was a Visiting Researcher at the Massachusetts Institute of Technology, Cambridge, MA, USA. His research interests include the control and robotics fields, in particular, intelligent systems. Prof. Hashimoto was the Vice-President of the IEEE Industrial Electronics Society (IES) and the Intelligent Transportation Systems Council (ITSC), and a Board Member of the Society of Instrument and Control Engineers of Japan. He initiated the IEEE/ASME International Conference on Advanced Intelligent Mechatronics in 1997. He is a Fellow of the IEEE, as well as the the Robotics Society of Japan, and the Society of Instrument and Control Engineers (SICE). 